\title{
Subtle Fluorination of Conjugated Molecules Enables Stable Nanoscale Assemblies on Metal Surfaces
}

\author{
Jens Niederhausen ${ }^{1,2,3}$, Yuan Zhang ${ }^{1}$, Fairoja Cheenicode Kabeer ${ }^{4}$, Yves Garmshausen ${ }^{5}$, Bernd M. \\ Schmidt ${ }^{6}$, Yang $\mathrm{Li}^{1}$, Kai-Felix Braun ${ }^{1}$, Stefan Hecht ${ }^{5}$, Alexandre Tkatchenko ${ }^{4,7}$, Norbert $\mathrm{Koch}^{2,3^{*}}$, and \\ Saw-Wai Hla ${ }^{1,8, *}$ \\ ${ }^{1}$ Nanoscale and Quantum Phenomena Institute, Physics \& Astronomy Department, Ohio University, \\ Athens, Ohio, 45701, USA. \\ ${ }^{2}$ Institut für Physik \& IRIS Adlershof, Humboldt-Universität zu Berlin, 12489 Berlin, Germany. \\ ${ }^{3}$ Helmholtz-Zentrum Berlin für Materialien und Energie GmbH, 12489 Berlin, Germany. \\ ${ }^{4}$ Fritz-Haber-Institut der Max-Planck-Gesellschaft, 14195 Berlin, Germany. \\ ${ }^{5}$ Institut für Chemie \& IRIS Adlershof, Humboldt-Universität zu Berlin, 12489 Berlin, Germany. \\ ${ }^{6}$ Institute of Organic and Macromolecular Chemistry, Heinrich-Heine-University Düsseldorf, 40225 \\ Düsseldorf, Germany \\ ${ }^{7}$ Physics and Materials Science Research Unit, University of Luxembourg, L-1511 Luxembourg \\ ${ }^{8}$ Center for Nanoscale Materials, Argonne National Laboratory, Argonne, IL, 60439, USA. \\ *Correspondence email: norbert.koch@physik.hu-berlin.de; hla@ohio.edu
}

\begin{abstract}
In molecular self-assembly on surfaces, the structure is governed by the intricate balance of attractive and repulsive forces between molecules as well as between molecules and substrate. Frequently, repulsive interactions between molecules adsorbed on a metal surface dominate in the low coverage regime, and dense self-assembled structures can only be observed close to full monolayer coverage. Here we demonstrate that fluorination at selected positions of conjugated molecules provides for sufficiently strong yet non-rigid $\mathrm{H} \cdots \mathrm{F}$ bonding capability that enables (i) the formation of stable nanoscale molecular assemblies on a metal surface and (ii) steering the assemblies' structure. This approach should be generally applicable and will facilitate the construction and study of individual nanoscale molecular assemblies with structures that are not attainable in the high coverage regime.
\end{abstract}


Molecular self-assembly is recognized as a key process in the formation of functional structures from the nano- to the macroscale in biology, chemistry, and physics. ${ }^{1-5}$ By exploiting the interplay of non-covalent interactions, complex and stable arrangements can be formed with minimal input of energy. The simultaneous action of attractive and repulsive forces cover a wide range of stabilizing and destabilizing energy values, from a few meV to several $100 \mathrm{meV} .{ }^{6}$ When selecting the appropriate magnitude for each intermolecular force in the target structure, already thermal energy at room temperature (ca. $26 \mathrm{meV}$ ) can provide for sufficient dynamics that enables efficient self-assembly and allows, e.g., for self-healing or adaptiveness to the environment of the molecular assembly. ${ }^{7}$

In the fields of organic and molecular electronics the self-assembly building blocks are predominantly $\pi$ conjugated molecules. By nature, these comprise intramolecular polar bonds. Therefore, electrostatic dipole and multipole interactions play an important role in structure formation. ${ }^{8,9}$ Likewise, hydrogen bonds can be a dominant factor in molecular self-assembly. ${ }^{10,11}$ In addition to these interactions of permanent charges, mutual polarization and dispersion interactions contribute substantially. All together are particularly important for molecules on solid surfaces such as metals, as the pairwise interaction magnitude may vary considerably for molecule-molecule and molecule-substrate. ${ }^{12,13}$ Yet, predicting the self-assembled structure for a given material combination, based on known molecular and substrate structure, remains one of the major challenges, as a reliable hierarchical ranking of the governing interaction energies based on empirical knowledge is not sufficiently possible.

In this contribution, we study the structure of assemblies formed by three conjugated molecules with very similar chemical structure, differing only by two fluorine atoms and the associated molecular dipole moment, on an $\operatorname{Ag}(111)$ surface. Scanning tunneling microscopy (STM) evidences that the self-assembled structures differ substantially, but attempts to explain these based on plausible consideration of the dominant interactions, dipole-dipole and fluorine-mediated hydrogen bonding, fail. Only with state-of-the-art density functional theory (DFT) modeling and deliberate discrimination of all involved interactions, i.e., 
electrostatic due to permanent charges, polarization and dispersion, as well as hydrogen bonding, we can fully rationalize the experimental findings and provide new insight for advanced self-assembly strategies. Our results underline the importance of very careful molecular design as numerous interaction parameters are correlated, but also demonstrate the distinctive power of modern DFT methods, developed only very recently, to provide understanding of fairly large and complex systems.

One approach to influence the structure of a molecular assembly is the introduction of a dipole in an otherwise non-dipolar molecule, in order to exploit dipole-dipole interaction. Frequently, this is achieved by replacing peripheral hydrogen of a conjugated molecule by electron withdrawing fluorine. A prototypical example that we consider here is para-sexiphenyl (6P) ${ }^{14-20}$ and two partially fluorinated derivatives (see Fig. 1a for chemical structures). For $0-2 \mathrm{~F}-6 \mathrm{P}$ two fluorine atoms are placed at the ortho position of a terminal benzene ring and for $m-2 \mathrm{~F}-6 \mathrm{P}$ at the meta position. The two highly polar C-F bonds in each molecule result in dipoles along the long axis, with opposite direction for the two molecules. However, this is already a simplistic view of the net-dipole creating charge density patterns. In the meta position, electron density is accumulated at the outward-directed fluorine atoms with negligible charge transfer from the neighboring benzene ring, yielding an electric dipole of 2.2 Debye in the gas phase (Fig. 1b). In contrast, for ortho substitution also the neighboring benzene ring contributes to the electron density accumulated at the fluorine atoms. This charge pattern can be viewed as two electric dipoles opposing each other, however, with different magnitude, yielding a smaller net-dipole moment of 1.1 Debye (Fig. 1c). These rather subtle differences already make it less obvious how the molecules would arrange on a metal surface. In addition, fluoroaromatics can feature $\mathrm{H} \cdots \mathrm{F}$ hydrogen bonding, whose stabilizing energy can vary vastly. ${ }^{21-26}$ In fact, replacement of hydrogen atoms by fluorine atoms has been shown to result in substantial changes in the bulk structure of conjugated molecules and polymers, with considerable impact on optical and charge transport properties. ${ }^{27-32}$ 

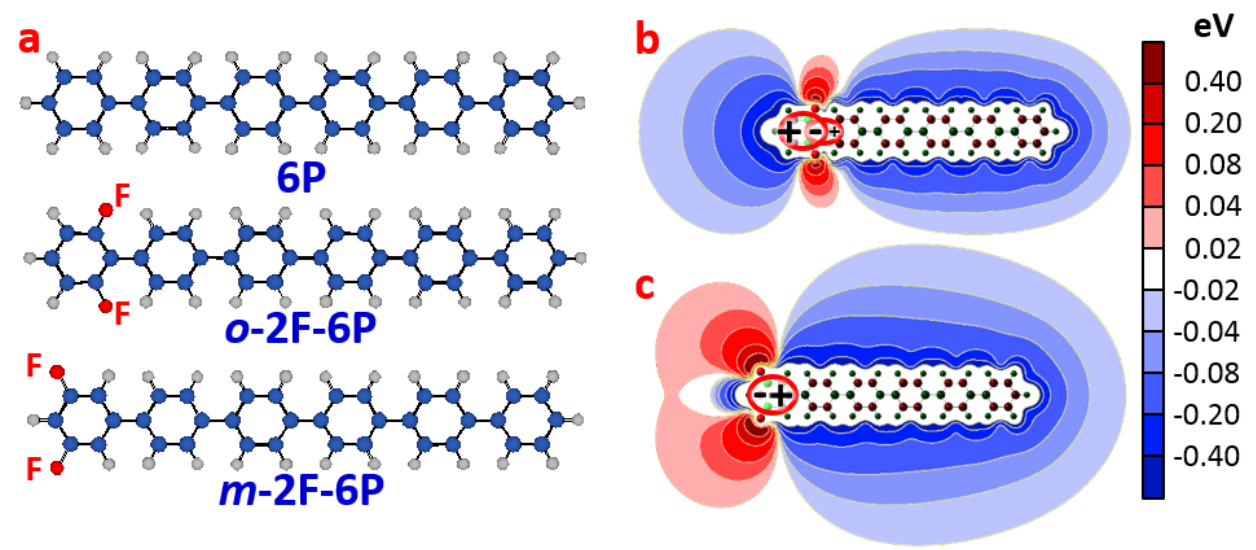

Figure 1. The employed molecules. (a) Chemical structure of 6P, $m-2 \mathrm{~F}-6 \mathrm{P}$, and $o-2 \mathrm{~F}-6 \mathrm{P}$. (b) and (c) The electrostatic potential maps of $o-2 \mathrm{~F}-6 \mathrm{P}$ and $m-2 \mathrm{~F}-6 \mathrm{P}$, respectively.

\section{Methods}

\section{General synthetic and analytical methods}

The intermediate building block 1-(4-bromophenyl)-4-(biphenyl-4-yl)-1,4bis(methoxymethyloxy)cyclohexane was described earlier. ${ }^{33}$ The syntheses of the additional building blocks 3,5-Difluoro-4'-(4,4,5,5-tetramethyl-1,3,2-dioxaborolan-2-yl)-biphenyl and 3,5-Difluorobiphenyl-4'boronic acid as well as the final products $o-2 \mathrm{~F}-6 \mathrm{P}$ and $m-2 \mathrm{~F}-6 \mathrm{P}$ are described in the Supplementary Information. ${ }^{1} \mathrm{H}$ and ${ }^{13} \mathrm{C}$ NMR spectra of intermediates and precursors can be also found there.

\section{Low temperature scanning tunneling microscopy}

The experiments were performed by using a custom-built ultrahigh vacuum (STM) system. ${ }^{34}$ A single crystal Ag(111) substrate was cleaned by repeated cycles of sputtering with Ne ions followed by annealing to 700K. For a comparative study, 6P and the two types of fluorinated 6P molecules were separately stepwise deposited by using the same deposition parameters from a custom-built Knudsen cell onto an $\operatorname{Ag}(111)$ substrate via thermal evaporation. Depositions of $m$-2F-6P were performed both with the sample at ca. 70 $\mathrm{K}$ and at room temperature without notable differences in the observed arrangement. Annealing of the molecular film did also not give rise to qualitative changes in the arrangements. Grain size and/or length of the supramolecular chains might very well be influenced by the preparation conditions, but such a 
quantitative analysis was prevented in the current experiments by the tip-molecule interaction that gave rise to frequent but random destruction of the molecular arrangements. O-2F-6P depositions were performed with the sample at room temperature while $6 \mathrm{P}$ was deposited on the sample at ca. $70 \mathrm{~K}$ substrate temperature. After each deposition, the sample was transferred to the STM chamber in-situ and cooled down to $\approx 6 \mathrm{~K}$ for the experiments. All presented images are representative for the respective molecular films as deduced from multiple film preparations and tens of different investigated spots per system.

A Gaussian or low pass filter were applied to some STM images to reduce the noise level. Some images are presented as rendered 3D view, as indicated in the figure caption.

\section{First-principles electronic structure calculations}

First-principles electronic structure calculations employed the DFT code FHI-aims ${ }^{35}$ to investigate the selfassembly of 6P molecules and fluorinated 6P derivatives adsorbed on the Ag (111) surface. All calculations have been performed with either the DFT+vdW method ${ }^{36,37}$ or the DFT+MBD method ${ }^{38}$ to account for vdW interactions. The DFT+vdW method combines the Tkatchenko-Scheffler (TS) DFT+vdW ${ }^{36}$ method for intermolecular interactions with the Lifshitz-Zaremba-Kohn (LZK) theory, ${ }^{36,39}$ including the collective response of the substrate electrons in the calculation of the molecule-surface vdW energy. The DFT+MBD method in addition includes many-body contributions to the vdW dispersion energy for both moleculesurface and molecule-molecule interactions. ${ }^{38,40}$ Throughout this work, we employ the Perdew-BurkeErnzerhof ${ }^{41}$ (PBE) functional with converged settings for the integration grids and standard numerical atomcentered orbitals basis sets. The atomic zeroth-order regular approximation (ZORA) ${ }^{42}$ was used to treat the relativistic effects for all atoms. A silver slab with $20 \times 8$ surface cell and 4 metal layers was used throughout. We used a Monkhorst-Pack grid of $1 \times 1 \times 1$ k-points. ${ }^{43}$

We have calculated the total energy for 6P dimer and fluorinated 6P dimer structures in the gas phase and on the $\mathrm{Ag}(111)$ surface, including dispersion interactions with both the DFT+vdW and DFT+MBD methods. The calculations were carried out for different stacking of the dimers as shown in Fig. 5 with the inter-ring twist angle of $\approx 11.4^{\circ} .^{18}$ The long molecular axes of the two molecules in the dimer structures are separated by $\approx 7.0 \AA,{ }^{20}$ resulting in a $\mathrm{H} \cdots \mathrm{F}$ contact length of ca. $2.6 \AA$. For the structures including the Ag surface, the 
molecules are located $\approx 3.0 \AA$ from the topmost metallic layer, ${ }^{44,45}$ and aligned along the Ag[110] direction. A visualization of the on-surface structures can be found in Supplementary Figure S2.

Dimer structures were not further optimized, but we tested the difference between relaxed and single point calculations. As reported in Supplementary Table S1, only the total energies differ notably, while the absolute binding energies, van der Waals contribution, and Hirshfeld charge transfer are comparable. Note that we report only relative binding energies in the manuscript. For these, the difference between relaxed and single point calculations are significantly reduced compared to the absolute values.

\section{X-ray diffraction}

To test the ability of our molecules in forming $\mathrm{H} \cdots \mathrm{F}$ hydrogen bonds, we attempted to grow single crystals. Sexiphenyls as used herein are generally arduous to crystallize, due to their low symmetry. We took great effort and succeeded in crystallizing a suitable model compound, $m-4 \mathrm{~F}-6 \mathrm{P},{ }^{27}$ by sublimation in a custommade apparatus $^{46}$ at high temperature over several days. All tested crystals showed twinning phenomena, which could be solved for one particularly good crystal and the data is presented in the Supplementary Information.

\section{Results and discussions}

While the changes in chemical structure of these three molecules may seem small, the self-assembled structure on $\mathrm{Ag}(111)$ in the submonolayer and monolayer regimes are huge. For the non-dipolar 6P we find individually adsorbed molecules at low coverage (see STM images in Fig. 2), and only when approaching monolayer coverage the formation of rows of uniform width formed by densely packed molecules with the long molecular axis perpendicular to the row direction (see Supplementary Fig. S21). 

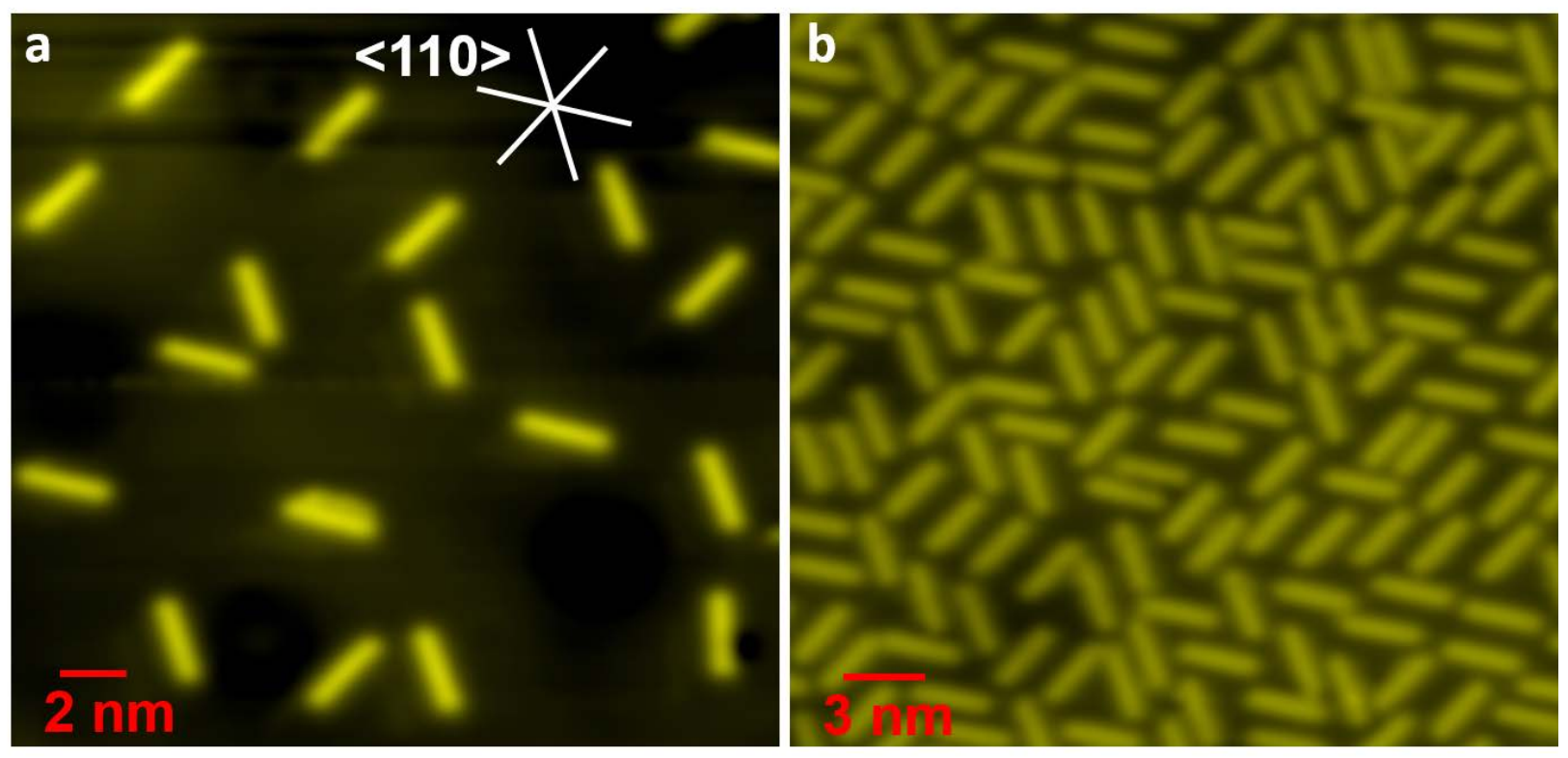

Figure 2. 6P structures. STM image of individual 6P molecules on $\mathrm{Ag}(111)$ (a) at low and (b) at medium coverage. The molecules are mostly oriented with their long molecular axis along [110] and equivalent directions of the surface. [a: $\mathrm{V}=-2 \mathrm{~V}, \mathrm{I}=0.1 \mathrm{nA}, 23.1 \mathrm{~nm}$ x $22 \mathrm{~nm}$ scan; b: $2 \mathrm{~V}, \mathrm{I}=27 \mathrm{pA} 28.1 \mathrm{~nm} \times 26.8$ nm scan].

For $o-2 \mathrm{~F}-6 \mathrm{P}$ we find only very few individual molecules on the surface. Most molecules assemble in rows as those shown in Fig. 3. The two consecutively taken images shown as insets of Fig. 3a evidence that molecules stay close-packed even when moved away from the row by the STM tip. In contrast to the 6P rows at high coverage, the $o-2 \mathrm{~F}-6 \mathrm{P}$ long molecular axes are not perfectly perpendicular to the row direction, and the edges of each row appear fringed, i.e., a stack-shift of one or two benzene rings between subsequent molecules is typical, notably, also in a configuration for which the ring that carries the proton acceptor for hypothetical $\mathrm{H} \cdots$ F hydrogen bond formation is overlapping with only one neighboring molecule. For a single o-2F-6P molecule, one end of the molecule appears brighter in STM imaging, which we attribute to the fluorine-bearing terminal benzene ring (indicated by the green arrow in Fig. 3b). The apparent height line profiles across twelve consecutive molecules within a row, as indicated by the alternating green and blue 
lines in Fig. 3b, are shown in Fig. 3c after removal of the stack-shift. This evaluation reveals alternating left and right higher ends of the molecules, evidencing that the molecular dipoles are positioned in an antiparallel arrangement, yet with quite large stack shifts (mostly more than two and routinely five benzene rings separating the dipole bearing terminal rings of two adjacent molecules).
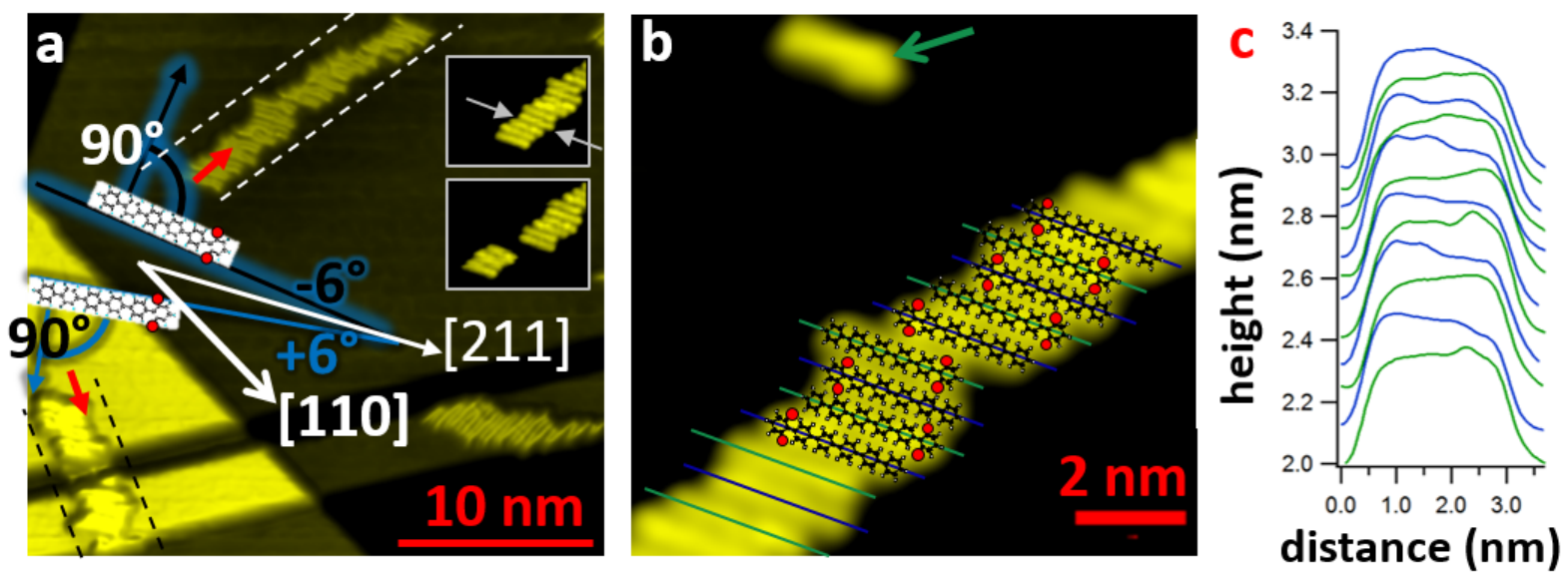

Figure 3. $\boldsymbol{O}-2 \mathrm{~F}-6 \mathrm{P}$ structures. (a) Large area STM image showing three self-assembled rows of $o-2 \mathrm{~F}-6 \mathrm{P}$ molecules. The bottom two rows exhibit a "carpet mode" where the molecules within a row cross over the surface steps and troughs. This indicates that the molecule-molecule interaction is sufficiently strong to overcome step-barriers. The molecular alignment and average stacking direction are indicated $[\mathrm{V}=-1.5 \mathrm{~V}$, $\mathrm{I}=0.1 \mathrm{nA}, 30 \mathrm{~nm} \times 28 \mathrm{~nm}$ scan, 3D view]. Inset: Two consecutively acquired images that evidence the STM scan-induced detachment of three hydrogen-bonded molecules at the indicated position $[\mathrm{V}=-1.5 \mathrm{~V}, \mathrm{I}$ $=0.1 \mathrm{nA}, 9.8 \mathrm{~nm}$ x $7.3 \mathrm{~nm} \mathrm{scan].} \mathrm{(b)} \mathrm{Molecules} \mathrm{form} \mathrm{a} \mathrm{stack} \mathrm{arrangement} \mathrm{with} \mathrm{lateral} \mathrm{shift} \mathrm{in} \mathrm{the} \mathrm{row.} \mathrm{A}$ single molecule appears with a bright end indicated with an arrow [V = -1.5V, I = $0.1 \mathrm{nA}, 11.4 \mathrm{~nm} \times 11.1$ nm scan]. (c) A sequence of line profiles of 12 consecutive molecules in (b) show higher heights at either right (green) or left (blue) ends. 

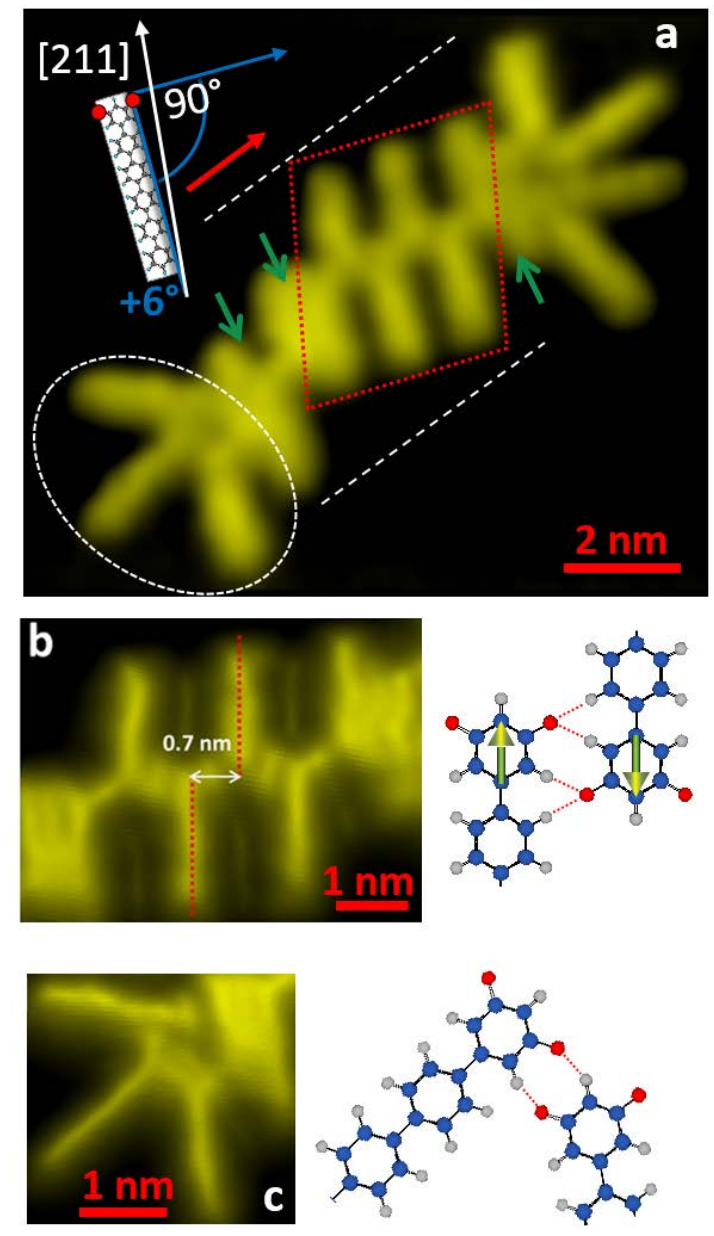

Figure 4. M-2F-6P structures. (a) STM image of $m$-2F-6P rows showing zipper (red dotted parallelogram) and canted (white dashed oval) arrangements. The green arrows indicate more densely packed local structures. The molecular alignment and average stacking direction are indicated $[\mathrm{V}=-1 \mathrm{~V}, \mathrm{I}=0.1 \mathrm{nA}, 15.3$ $\mathrm{nm} \times 9.4 \mathrm{~nm}$ scan]. (b) A zoom of a zipper arrangement [V = -1V, I = $0.1 \mathrm{nA}$, 3D view]. The measured distance between the neighboring molecules is $0.7 \mathrm{~nm}$. The corresponding schematic shows antiparallel dipole configuration and $\mathrm{H} \cdots \mathrm{F}$ bonding sites. (c) STM image of a canted arrangement $[\mathrm{V}=-1 \mathrm{~V}, \mathrm{I}=0.1 \mathrm{nA}$, 3D view]. The corresponding schematic shows $\mathrm{H} \cdots \mathrm{F}$ bonding sites. Since the canted arrangement facilitates a 'turnaround' of the supramolecular chain, it allows all fluorine atoms to feature an $\mathrm{H} \cdots \mathrm{F}$ bond. 
Finally, the structure formed by $m$-2F-6P differs distinctly from the former two. A line profile analysis like the one in Fig. 3c yielded also antiparallel adjacent molecules (c.f. Supplementary Fig. S24). However, the stack-shift between subsequent molecules is huge, very often five benzene rings, resulting in the apparent “zipper” structure seen in Fig. 4. In some instances, deviations from this zipper configuration are observed. For instance, the green arrows in Fig. 4a indicate such regions where neighboring molecules have a smaller stack-shift. Similar to the case of $o-2 F-6 P$, such regions give rise to a significant discrepancy between the orthogonal of the long molecular axes and the long-range stacking direction. As for $0-2 \mathrm{~F}-6 \mathrm{P}$ and for $6 \mathrm{P}$ rows at high coverage, the distance between subsequent molecules is $0.7 \mathrm{~nm}$. This was shown in a previous STM study to correspond to flat-on adsorbed molecules, while a distance of $0.4 \mathrm{~nm}$ would be tantamount for an edge-on adsorption geometry. ${ }^{20}$ At the end of rows, we often find a "canted" arrangement of $m$-2F-6P with angles of ca. $60^{\circ}$ between the molecular axes, thus forming a "star-like" end of a row. As the side-by-side arrangement allows for the maximum dipole-dipole effect and, in view of the extreme polarizability asymmetry of rod-like molecules, also gives rise to the largest dipole-induced dipole effect, the observation of the canted arrangements is first evidence that the molecular dipole moment by itself does not dominate the structures.

The fact that we find individual 6P molecules on $\mathrm{Ag}(111)$ and, instead, molecular aggregates for the fluorinated ones at low coverage, indicates that repulsive interactions dominate between 6Ps, while net attractive forces persist for adsorbed $o-2 \mathrm{~F}-6 \mathrm{P}$ and $m-2 \mathrm{~F}-6 \mathrm{P}$ molecules. At first sight, the interaction of molecular dipoles and $\mathrm{H} \cdots \mathrm{F}$ hydrogen bonds would appear as candidates facilitating aggregate formation.

However, without further insight it remains elusive why apparently both interactions dictate the structure for m-2F-6P (immediate proximity of molecular dipoles and possibly two $\mathrm{H} \cdots \mathrm{F}$ bonds per molecule), but for $0-2 \mathrm{~F}-6 \mathrm{P}$ a configuration with seemingly non-optimal stack-shift for less than two $\mathrm{H} \cdots \mathrm{F}$ bond formation persists and the dipoles are generally rather separated in space. Furthermore, the molecular packing density 
varies significantly in the different row arrangements, so that also stabilizing polarization and dispersion interactions vary substantially as well.

To gain the required insight, we investigate the binding energy of molecular dimers as a function of lateral shift between them, i.e., the stack-shift, with DFT using the PBE functional. One of the molecules is kept in a fixed position, while the other molecule is shifted (one ring at a time, for nomenclature of the different arrangements, see Fig. 5) along the long molecular axis direction. Furthermore, to single out individual interaction type contributions, we compare the dimers without and with the $\mathrm{Ag}(111)$ substrate, and we omit (pure PBE calculations) or include van der Waals (vdW) interactions intentionally. The latter is done by employing a pairwise Tkatchenko-Scheffler vdW model ${ }^{36}$ and also the more sophisticated many body dispersion (MBD) method. ${ }^{38}$

However, to justify a comparison between experiment and theory, we first have to make sure that the observed assemblies correspond to equilibrium structures, i.e., are not kinetically frustrated.

As shown in Supplementary Fig. S21a, the kinetic energy of 6P deposited onto substrates at ca. $70 \mathrm{~K}$ is sufficient for the molecules to align along the $\mathrm{Ag}[110]$ direction, thereby overcoming the rotational energy barriers associated with their very asymmetric shape. In addition, Supplementary Figure S21b evidences that densely packed and ordered monolayers form once surface energy minimization acts as driving force, further proof that the kinetic energy is indeed sufficient to find the energetic minimum. The 6P molecules that are shown in Fig. 2 were deposited onto the substrate held at room temperature, additionally guaranteeing that no kinetic barriers influence the molecular self-assembly. 


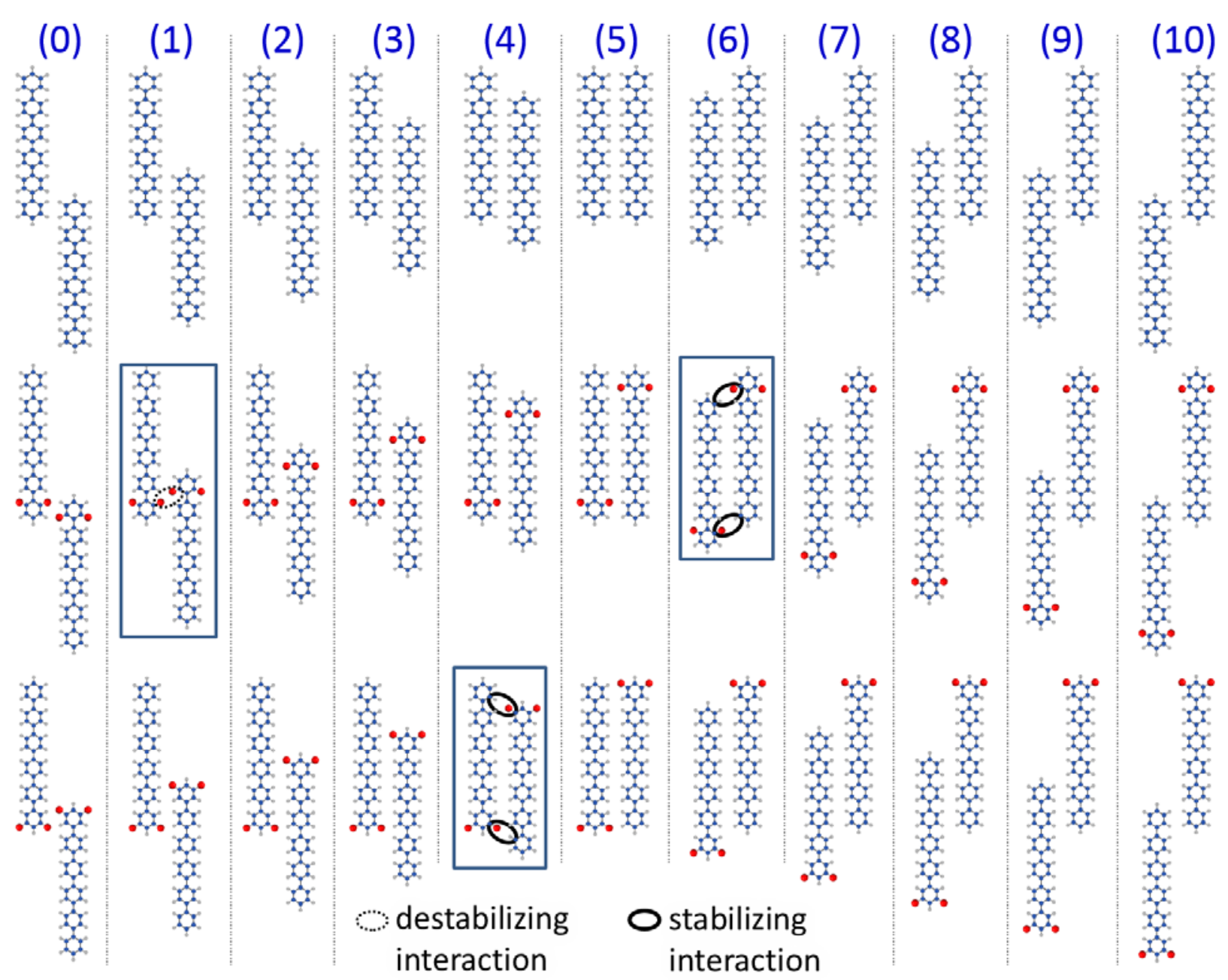

Figure 5. The dimer structures used for the calculations. The boxes indicate configurations that highlight specific interactions (encircled) between rings that are not direct neighbors as discussed in the main text. The intermolecular F to F contact in the doted oval amounts to $2.9 \AA$. 

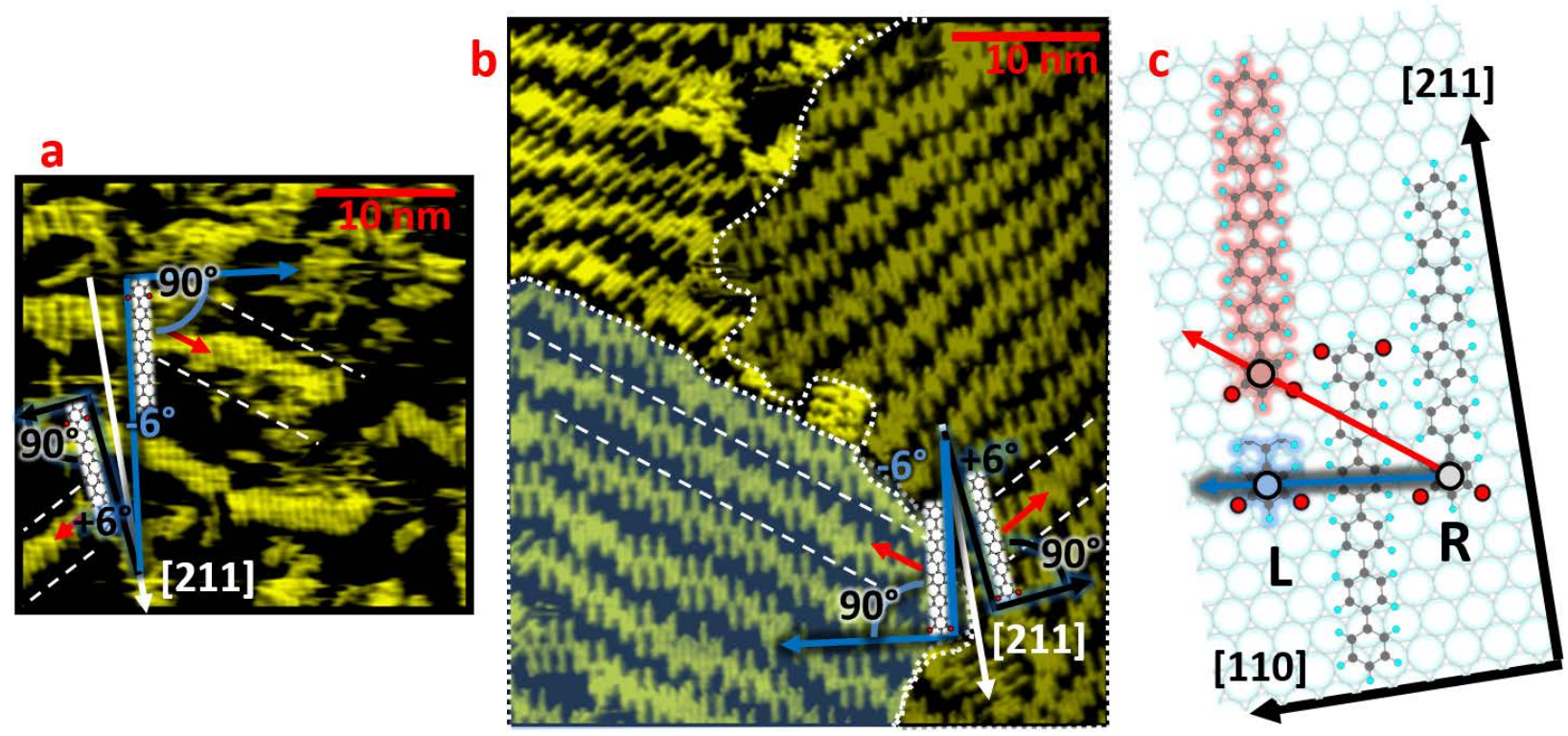

Figure 6. Oriented supramolecular chains at higher coverage. (a) and (b) Large area STM images that show self-assembled rows of $o-2 \mathrm{~F}-6 \mathrm{P}$ and $m-2 \mathrm{~F}-6 \mathrm{P}$ molecules on $\mathrm{Ag}(111)$, respectively. The blue and black lines identify the two surface enatiomers. [a: $\mathrm{V}=1 \mathrm{~V}, \mathrm{I}=0.3 \mathrm{nA}, 29.8 \mathrm{~nm}$ x $28.4 \mathrm{~nm}$ scan; b: $\mathrm{V}=1 \mathrm{~V}, \mathrm{I}=$ $0.3 \mathrm{nA}, 47 \mathrm{~nm} x 45 \mathrm{~nm}$ scan]. (c) Illustration (for m-2F-6P) showing non-equal epitaxial registries for the molecule on the left (L) and right $(\mathrm{R})$ of a given molecule.

To see whether the stack-shift of the $m-2 \mathrm{~F}-6 \mathrm{P}$ and $o-2 \mathrm{~F}-6 \mathrm{P}$ assemblies correspond to their equilibrium state, we take a closer look at their long-range stacking direction. Figures $6 \mathrm{a}$ and $\mathrm{b}$ depict $m-2 \mathrm{~F}-6 \mathrm{P}$ and $o-2 \mathrm{~F}-6 \mathrm{P}$ on $\operatorname{Ag}(111)$, respectively, with higher coverage than those shown in Figs. 3 and 4. As mentioned above, the stacking directions along the supramolecular chains (indicated by the red arrows and white dashed lines) clearly deviate from assumed stacking perpendicular to the long molecular axes (represented by the blueand-black arrows in the same figures). This deviation is a macroscopic manifestation of different stack-shift distributions at the two sides of a given molecule. This can be attributed to the fact that the long molecular axes and the [211] ([110]) direction enclose an angle of ca. $\pm 6^{\circ}\left( \pm 24^{\circ}\right)$. For the molecules to the left and right of a given molecule in the row ( $\mathrm{L}$ and $\mathrm{R}$, respectively; see Fig. $6 \mathrm{c}$ for definition), this results in different epitaxial registries for assumed identical stack-shifts. Thus, the energetic landscapes on the two sides of a 
given molecules are slightly different, causing $\mathrm{L}$ and $\mathrm{R}$ molecules to attach with different average stack shifts. Being rotated by $180^{\circ}$, the inverted consideration applies for the following molecules within a row, in turn giving rise to the observed finite angle between stacking direction and the long molecular axes. In contrast, a long-range stack direction perpendicular to the molecular axes would be found for random attachment of molecules expected for kinetically frustrated molecular arrangements.

Substrate temperatures during deposition of ca. $70 \mathrm{~K}$ and room temperature, as well as post-deposition annealing, all resulted in the same stack-shift distribution for $m-2 \mathrm{~F}-6 \mathrm{P}$. This shows that all observed structures have equally reached their equilibrium configuration and that the variation in stack-shift along the supramolecular chains stems from entropic disorder. Long-range oriented supramolecular chains with entropic disorder were also observed for o-2F-6P deposited onto $A g(111)$ held at room temperature, evidence for an equilibrium situation also in this case. $M-2 \mathrm{~F}-6 \mathrm{P}$ primarily exhibits stack-shifts for $\mathrm{L}$ and $\mathrm{R}$ in the range of (0) to (5) and (0) to (2), respectively, while for most $o$-2F-6P molecules the respective stackshifts ranges are (4) - (6) and (2) - (5), resulting in similar stacking directions in the two cases.

We now turn towards the calculated energy curves as function of stack-shift. The results for 6P dimers are summarized in Fig. 7a and 7b, with the binding energy plotted with respect to the energy of the least stable structure. In the gas phase, PBE favors the least lateral proximity of the molecules, represented by structures (0) and (10) in our model. This is due to Pauli-repulsion, giving rise to the repulsive part of the intramolecular interaction potential, which is included in the standard PBE calculations. The inclusion of attractive intermolecular interactions via parameterized vdW forces and many-body dispersion in more realistic functionals ( $\mathrm{PBE}+\mathrm{vdW}$ and $\mathrm{PBE}+\mathrm{MBD}$, respectively) expectedly changes this trend and returns the side-by-side configuration (5) as the most stable arrangement of $6 \mathrm{P}$ in the gas phase. The dimer binding energy scales with the number of laterally overlapping benzene rings and is estimated from the slope of each branch of the V-shaped relations in Fig. 7a as ca. $45 \mathrm{meV}$ per ring pair. 


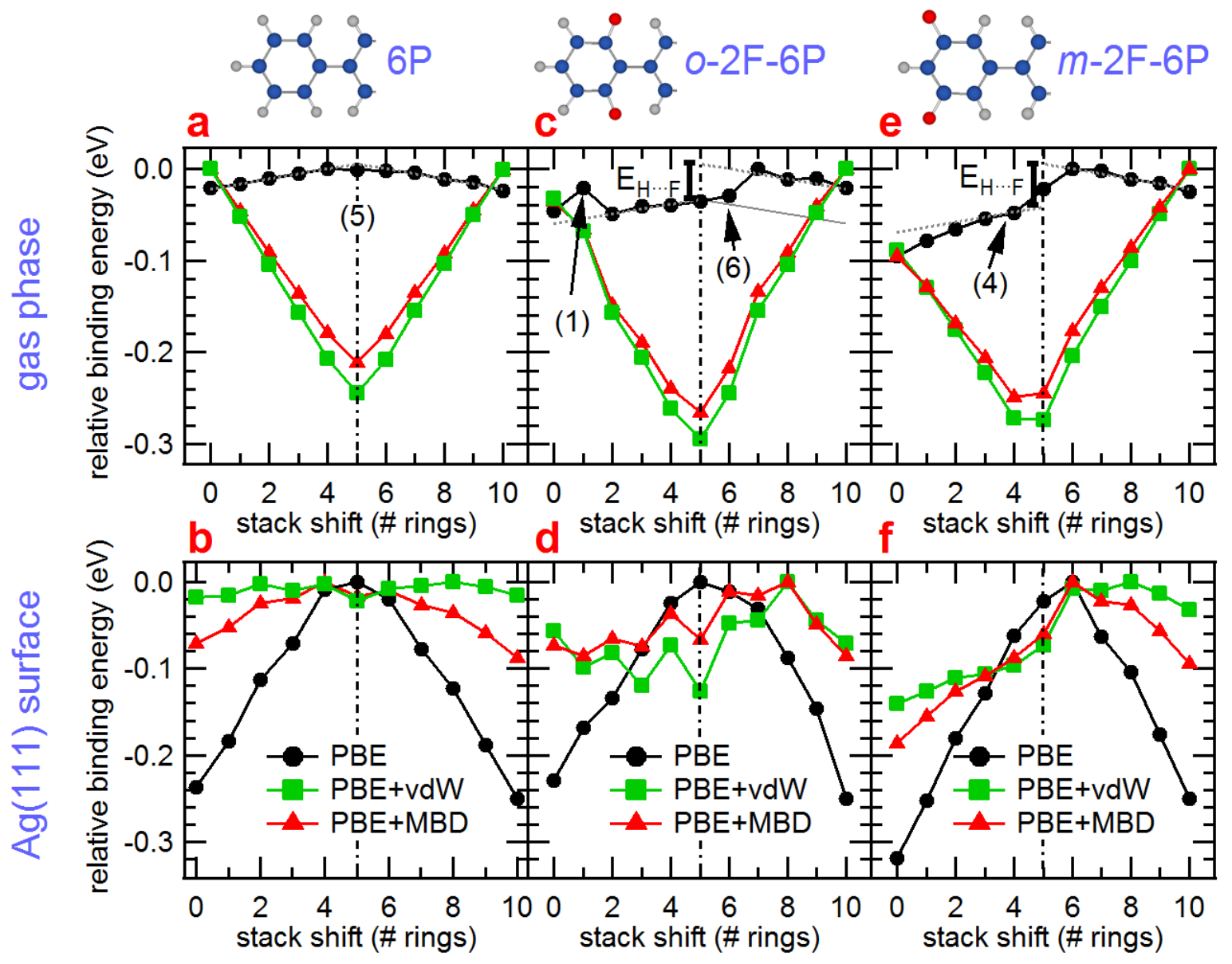

Figure 7. Calculated dimer binding energies. (a) Binding energy vs. lateral displacement (stack-shift) for gas phase 6P dimer arrangements calculated using PBE (black), PBE+vdW (green), and PBE+MBD (red) methods. The binding energy of each dimer is plotted with respect to the energy of the least stable structure.

(b) The same as (a) but with dimer structures on Ag(111) surface. (c) and (d) The same as (a) and (b), respectively, but for o-2F-6P dimer arrangements. (e) and (f) The same as (a) and (b), respectively, but for m-2F-6P dimer arrangements. The error bars of the calculations themselves are ca. $3 \mathrm{meV}$, i.e., roughly represented by the symbol size. However, the adsorption geometries can only be approximately accounted for in these calculations, adding another uncertainty of ca. $20 \mathrm{meV}$ upon including the Ag(111) surface.”

The situation changes radically upon including the Ag(111) surface. A small but finite electron transfer of 0.04 electrons from the metal to each 6P molecule induces a dipolar electrostatic density distribution 
between the molecule and the surface. The charge transfer was computed using the Hirshfeld partitioning of the electron density. ${ }^{47}$ The transferred charge is the sum over atomic charges on the molecule (or, equivalently, the surface) computed from the self-consistent electron density of the combined molecule/surface system. Consequently, the interaction of equally oriented dipoles of neighboring molecules gives rise to repulsion, as expressed by the strong inverted V-shape of the PBE relation in Fig. 7b. Note that the charge will be distributed throughout the molecule in the former lowest unoccupied molecular orbital, meaning that a point dipole model will not represent the repulsion adequately. From the slope in Fig. 7b we estimate the repulsive energy per laterally overlapping benzene ring pair to be ca. 58 meV. Including polarization and dispersion via $\mathrm{PBE}+\mathrm{vdW}$ and $\mathrm{PBE}+\mathrm{MBD}$ calculations reduces the intermolecular repulsion significantly. As seen from the experimental observation (Fig. 2), molecular aggregation is still energetically not favored. Consistently, the interaction energies for on-surface 6P dimer formation in the PBE+MBD calculations are positive (c.f. Supplementary Table S2), pointing to a net destabilizing intermolecular interaction. In addition, at low coverages beyond-dipole correlation effects could provide an additional mechanism for repulsive interactions. ${ }^{48}$ Similar cases of intermolecular repulsion were observed previously in several studies including 6P on $\mathrm{Cu}(111)$ and para-quaterphenyl on $\mathrm{Au}(111),{ }^{19,49-56}$ and also the transition from individually adsorbed molecules at low coverage to densely packed assemblies at higher coverage. ${ }^{49-56}$ Dense packing goes in hand with reduced charge transfer per molecule, but becomes energetically favorable due to the minimization of the surface energy as molecules cover the metal surface (compare, e.g., Refs. 57 and 58).

The same type of dimer-based modeling was performed for $o$ - and $m$-2F-6P (Fig. 7c-f). For both molecules we find the same small electron transfer from the metal, implying that the repulsion of surface-normal dipoles persists, but we can now revert to the details of attractive interactions, which allow for aggregate formation in the low coverage regime as seen in experiment. First of all, from the dimer binding energy dependence on stack-shift obtained from PBE in the gas phase we can retrieve the strength of the H$\cdots \mathrm{F}$ bond for our fluoroaromatics. From Fig. 7c we observe linear trends for o-2F-6P when going from structure (2) 
to (6) and from (7) to (10). The slopes of these trends are representative of the Pauli-repulsion per laterally overlapping benzene rings, and equal to those found for $6 \mathrm{P}$ in Fig. 7a. Notably, there is an offset $\mathrm{E}_{\mathrm{H} \ldots \mathrm{F}}$ between the two lines when going from configuration (6) to (7), which coincides with either having the possibility of forming two $\mathrm{H} \cdots \mathrm{F}$ bonds per dimer or none. This means that hydrogen bonds are contributing significantly also for configuration (6) that has the fluorine-bearing terminal benzene ring without a direct neighboring benzene ring, rationalizing the observation of this configuration despite seemingly less perfect lateral overlap. A deviation from maximum hydrogen bond strength for this configuration is apparent from the difference between the corresponding binding energy and that of configuration (4), as visualized in Fig. 7c by means of the solid line. Conversely, configuration (0) lacks maximum stabilization since it only features hydrogen bonding to the neighboring ring but does not benefit from an additional interaction with a second ring. Fully analogous behavior is observed for $m-2 \mathrm{~F}-6 \mathrm{P}$, only the configurations where the transition from no to full hydrogen bond stabilization occurs are shifted to (4)/(6), while in this case configuration (5) is in between in terms of full $\mathrm{H} \cdots \mathrm{F}$ bond formation capability. Apparently, the intermolecular bonds formed by the fluorine atoms feature contributions from more than one hydrogen atom and involve up to two rings. In addition, Arras et al. concluded for an equivalent atomic configuration that the proton acceptor interacts with the whole benzene ring, including important contributions from the C-H groups on the far side. ${ }^{59}$ This means that the interaction at hand exceeds the local character of a typical hydrogen bond.

It is worthwhile noting that $\mathrm{E}_{\mathrm{H} \cdots \mathrm{F}}$ is identical for $m-2 \mathrm{~F}-6 \mathrm{P}$ and $o-2 \mathrm{~F}-6 \mathrm{P}$, while for any assumed effect of the molecular dipole via dipole-induced dipole interaction the step size should relate to the rather different dipole moments of the two molecules.

While here only stack-shifts by integer benzene rings were considered to warrant comparability between the configurations, maximum stabilization could occur for other shift values. A detailed calculation of noninteger stack-shifts (see Supplementary Fig. S1) allows us estimating the binding energy per $\mathrm{H} \cdots \mathrm{F}$ bond of 
up to $30 \mathrm{meV}$ (25 meV for integer-only stack-shifts) for the molecules considered here. The atomic configurations that correspond to minima in the binding energy curve - with the fluorine atom being located in between two hydrogen atoms of a benzene ring or in between two phenyl groups - agree with those found for 6P-dicarbonitrile/Ag(111) ${ }^{60}$ and also the experimentally observed stack-shifts (Figs. 3b and 4b).

Inspection of Fig. 7c reveals that also configuration (1) for o-2F-6P is destabilized in energy compared to the straight line. This is attributed to the strong repulsion of two highly polar C-F bonds facing each other. For $m-2 F-6 P$, the slope of the left branch (see Fig. 7e) is found to be steeper than for the other two molecules. We believe that this is due to the two intramolecular dipoles coming into an energy-minimized antiparallel arrangement, leading to additional stabilization of the (0) and (1) configuration. We note that an apparent contribution to the dimer structure from intramolecular dipole interactions are missing for $0-2 \mathrm{~F}-6 \mathrm{P}$, which we ascribe to the smaller overall dipole moment and the fact that the electric potential distribution in the near field (the one relevant for two molecules in proximity) as shown in Fig. 1b deviates strongly from a simple dipolar pattern.

By including vdW interactions via PBE+vdW and PBE+MBD calculations (Figs. 7c and 7e) the trends for gas phase dimers changes towards the V-shape already observed for 6P, with an energy minimum at or near configuration (5). As this configuration was not observed in experiment for $o$ - and $m-2 \mathrm{~F}-6 \mathrm{P}$, the metal substrate must play a key role. In pure PBE calculations the surface-normal dipole-dipole repulsion, induced by the metal-to-molecule electron transfer, dominates and results in the distinct reversed V-shape (Figs. 7d and 7f). For $o-2 \mathrm{~F}-6 \mathrm{P}$, including vdW and MBD interactions lets a range of configurations becoming very similar in energy. The reduced structural symmetry upon including the substrate gives rise to more pronounced fluctuations in the calculated energy curves. When we bear in mind that non-integer stack-shifts can facilitate stronger hydrogen bonds, we see that configurations (2) to (7) are plausible, which we indeed find in experiment (Fig. 3). Apparently, the formation of $\mathrm{H} \cdots \mathrm{F}$ bonds, in combination with the polarization and dispersion interactions brought about by the substantial lateral overlap of neighboring molecules, is 
sufficient to overcome the charge transfer induced dipole repulsion, and aggregation of $o-2 \mathrm{~F}-6 \mathrm{P}$ molecules to rows with small stack-shifts can occur at very low absolute substrate coverage. The fact that the calculations reveal no clear energy minimum is reflected in the observation of several different stack-shifts in Fig. 3.

Configurations (0) and (1) are not found for $o-2 \mathrm{~F}-6 \mathrm{P}$, as the $\mathrm{H} \cdots \mathrm{F}$ bonds are weak or even antibonding. Configurations without $\mathrm{H} \cdots \mathrm{F}$ bonds are unstable as well. As already discussed for the gas phase dimers above, the interaction of intramolecular dipoles (i.e., in-plane) does not contribute notably to the assembly energy.

For $m-2 F-6 P$, the energy minimum is found for configuration (0) (see Fig. 7f). As this is the configuration we also find in experiment (Fig. 4), we conclude that here the $\mathrm{H} \cdots \mathrm{F}$ bonds and the (comparably smaller) intramolecular dipole interaction (crudely estimated in a point dipole model to be $11 \mathrm{meV}$ ) are overall stabilizing, while the small lateral overlap between direct neighbor molecules reduces polarization and dispersion related stabilization. However, this seems compensated for by the wider separation of destabilizing surface-normal dipoles in the configurations with large stack-shifts. Like for $o-2 \mathrm{~F}-6 \mathrm{P}$, the relatively flat energy curves for calculations that assume m-2F-6P molecules aligned with [110] will be slightly altered by the actual mismatch of long molecular axes and the Ag high symmetry directions. This can explain why configuration (0) dominates predominantly on one side of a given $m$-2F-6P molecule, while its other side sometimes exhibits smaller stack-shifts. From a comparison of the binding energies of configurations (0) and (10) for $m-2 \mathrm{~F}-6 \mathrm{P}$ we can estimate the combined dimer stabilizing energy due to two $\mathrm{H} \cdots \mathrm{F}$ bonds and the in-plane dipole arrangement to be ca. $100 \mathrm{meV}$.

Similar energetic considerations apply for the appearance of the canted arrangements found for $m-2 \mathrm{~F}-6 \mathrm{P}$, typically at the end of rows (Fig. 4c). In contrast to o-2F-6P, the meta-position of the fluorine atoms allows the formation of hydrogen bonds between two meta-2F-6P molecules also when the molecules "dock" with 
their heads while their long molecular axes form an angle of $\approx 60^{\circ}$ with neighboring molecules (Fig. 4c), a supramolecular building block (synthon) also found for 1,4-difluorobenzene, 1,3,5-trifluorobenzene, and 1,2,3,4-tetrafluorobenzene crystals. ${ }^{61,62}$ Noteworthy, several docking arrangements appear similarly stable. This is apparent in Fig. 4a from the different configurations at the two ends of the molecular row. In addition, manipulation with the STM tip induces the docking directions of molecules in canted arrangements to change repeatedly through a variety of configurations, as reported in the Supplementary Fig. S23. This corresponds to a wide range of possible $\mathrm{C}-\mathrm{F} \cdots \mathrm{H}-\mathrm{C}$ angles that all result in hydrogen bond formation, consistent with the rather flat interaction potential map between proton acceptors and organic rings found in Ref. 59 and evidence that employing this geometric parameter for the identification of hydrogen bonds, as typically done in crystal structure analysis, ${ }^{21-24}$ might not be appropriate for the $\mathrm{H} \cdots \mathrm{F}$ connected structures investigated here. For comparison, we also studied $m-4 \mathrm{~F}-6 \mathrm{P}$, a symmetric congener of $m$-2F-6P, in the solid state by means of single-crystal X-ray analysis, where the existence of significant classic $\mathrm{H} \cdots \mathrm{F}$ bonds can be observed via short to medium intermolecular $\mathrm{H} \cdots \mathrm{F}$ contacts ${ }^{61,62}\left(2.43-2.67 \AA\right.$ with angles $143-175^{\circ}$, c.f. Supplementary Table S3), leaving little doubt that these weak interactions govern the overall structural alignment, at least to some extent.

\section{Conclusions}

In summary, we obtained detailed insight into the intricate interplay of attractive and repulsive interactions that govern the self-assembly of molecules on a metal surface. Three structurally very similar conjugated molecules, i.e., $6 \mathrm{P}$ and the analogues $o-2 \mathrm{~F}-6 \mathrm{P}$ and $m-2 \mathrm{~F}-6 \mathrm{P}$ with two fluorine atoms each, showed vastly different assembly phenomenology on $\mathrm{Ag}(111)$. A satisfactory explanation of the experimental observations based on known molecular properties, such as intramolecular dipole moment, and presumed intermolecular interactions, such as $\mathrm{H} \cdots \mathrm{F}$ bond formation, could not be obtained, as a hierarchical ordering of all involved interactions and their coaction is not reliably possible. Only by employing a range of DFT methods, we could understand that the strongest repulsive component in all three systems is the Coulombic repulsion resulting from minute electron transfer from the metal to each molecule. Of comparable magnitude but of 
attractive character are intermolecular van der Waals interactions for densely packed molecules, i.e., polarization and dispersion. Yet, for 6P repulsion dominates and only isolated adsorbed molecules persist in the low coverage regime, and assembled structures only form close to monolayer coverage. For the partially fluorinated molecules, the $\mathrm{H} \cdots \mathrm{F}$ bond formation propensity turns out as the key factor enabling the formation of stable nanoscale molecular assemblies at very low absolute molecular coverage. Due to the small magnitude of $o-2 \mathrm{~F}-6 \mathrm{P}$ 's dipole moment $(1.1 \mathrm{D})$ and the pronounced deviation from a dipole charge pattern in the near field, the influence of this intramolecular dipole on the assembly structure was found to be negligible. In contrast, for $m-2 \mathrm{~F}-6 \mathrm{P}$, the charge pattern causing the intramolecular dipole (2.2 D) facilitates a small stabilizing dipole-dipole interaction. In conjunction with geometry-optimized $\mathrm{H} \cdots \mathrm{F}$ bonding between $m-2 \mathrm{~F}-6 \mathrm{P}$ molecules, contributing a binding energy of up to $30 \mathrm{meV}$ per bond, zipper-like rows form. This assembly, however, does not feature the dense packing observed for $o-2 \mathrm{~F}-6 \mathrm{P}$, as the Coulomb repulsion between the fluorine atoms in ortho configuration prevents the fully stabilized hydrogen bonds of a zipper-like configuration. Only with this detailed understanding of all interactions and their interplay it becomes feasible to design molecules for achieving targeted self-assembly structures, based on weak interactions only. Importantly, we illustrate ways how to circumvent dominating repulsive interactions at very low molecular surface coverage. This enables the realization of individual and stable nanoscale molecular assemblies and their study, without the need to approach monolayer coverage, which could substantially alter the obtained structure. Furthermore, the insight provided here helps understanding how fluorine substitution in conjugated molecules and polymers contributes to thin film and bulk structures, which, in turn, will enable realizing organic electronic materials with superior optical and charge transport properties for electronic and optoelectronic applications.

\section{Supporting Information}

Contents of the material supplied as Supporting Information: First-principles electronic structure calculations: additional calculations for dimers with non-integer stack shift, visualization of on-surface structures, relaxed vs. single point calculations, interaction energies for 6P dimer formation on $\mathrm{Ag}(111)$; 
synthetic and analytical methods: ${ }^{1} \mathrm{H}$ and ${ }^{13} \mathrm{C}$ NMR spectra of intermediates and precursors as well as IR spectra of the final compounds and 6P; additional scanning tunneling data: molecular arrangement of 6P in the monolayer regime, detailed discussion of $o-2 \mathrm{~F}-6 \mathrm{P}$ and $m-2 \mathrm{~F}-6 \mathrm{P}$ arrangements, scanning tunneling spectroscopy results for $m-2 \mathrm{~F}-6 \mathrm{P}$, manipulation of a canted arrangement with the STM tip, line profiles for $m-2 \mathrm{~F}-6 \mathrm{P}$; crystal structure of $m-4 \mathrm{~F}-6 \mathrm{P}$.

\section{Acknowledgements}

We acknowledge financial support by the US Department of Energy, Office of Science, Office of Basic Energy Sciences grant, DE-FG02-02ER46012 and by the DFG (SFB951). J.N. and N.K. acknowledge a German Academic Exchange Service (DAAD) award. J. N. thanks Georg Heimel for fruitful discussions. Use of the Center for Nanoscale Materials, an Office of Science user facility, was supported by the US Department of Energy, Office of Science, Office of Basic Energy Sciences, under contract no. DEAC02-06CH11357.

\section{Author Contributions}

J. N., N. K., and S-W. H conceived and designed the STM experiments and J. N., Y. Z., Y. L. and K.-F. B. performed the measurements. J. N., F. C. K, A. T., and N. K. conceived the DFT calculations and F. C. K performed them. Y. G. and S. H. conceived the synthesis and Y. G. synthesized the molecules, J. N., Y. Z., Y. L, F. C. K, and S-W.H. analyzed the data, B. M. S. performed and analyzed the X-ray diffraction experiments, J. N., N. K., and S-W. H co-wrote the paper with contributions from all other authors.

\section{Competing financial interests}

The authors declare no competing financial interests. 


\section{References}

[1] Whitesides, G. M.; Grzybowski, B. Self-Assembly at All Scales. Science 2002, 295, 2418-2421.

[2] Packwood, P.; Han, T.; Hitosugi, T. Chemical and Entropic Control on the Molecular Self-Assembly Process. Nat. Comm. 2017, 8, 14463.

[3] MacLeod, J. M.; Rosei, F. Molecular Self-Assembly on Graphene. small 2014, 10, 1038-1049.

[4] Lehn, L.M. Perspectives in Supramolecular Chemistry - From Molecular Recognition Towards Molecular Information Processing and Self-Organization. Ang. Chemie Int. Ed. 1990, 29, 1304-1319.

[5] Palmer, L.C.; Stupp, S. I. Molecular Self-Assembly Into One-Dimensional Nanostructures. Acc. Chem. Res. 2008, 41, 1674-1684.

[6] Supramolecular Materials for Opto-Electronics; Koch, N., Ed.; Royal Society of Chemistry, ISBN 978-1-84973-826-2, 2015; pp. 53-118.

[7] Wang, A.; Shi, W.; Huang, J.; Yan, Y. Adaptive Soft Molecular Self-Assemblies. Soft Matter 2016, 12, 337-357.

[8] Baber, A. E.; Jensen, S. C.; Sykes, E. C. H. Dipole-Driven Ferroelectric Assembly of Styrene on Au\{111\} J. Am. Chem. Soc. 2007, 129, 6368-6369.

[9] Niederhausen, J.; Kersell, H. R.; Christodoulou, C.; Heimel, G.; Wonneberger, H.; Müllen, K.; Rabe, J. P.; Hla, S.-W.; Koch, N. Monolayer Phases of a Dipolar Perylene Derivative on Au(111) and Surface Potential Build-Up in Multilayers Langmuir 2016, 32, 3587-3600.

[10] Slater, A. G.; Perdigão, L. M. A.; Beton, P. H.; Champness, N. R. Surface-Based Supramolecular Chemistry Using Hydrogen Bonds. Acc. Chem. Res. 2014, 47, 3417-3427.

[11] Barth, J. V.; Weckesser, J.; Cai, C.; Günter, P.; Bürgi, L.; Jeandupeux, O.; Kern, K Building Supramolecular Nanostructures at Surfaces by Hydrogen Bonding. Angew. Chem.; Int. Ed. 2000, 39, 1230.

[12] Liu, W.; Tkatchenko, A.; Scheffler, M. Modeling Adsorption and Reactions of Organic Molecules at Metal Surfaces. Acc. Chem. Res. 2014, 47, 3369-3377. 
[13] Maurer, R. J.; Ruiz, V.G.; Camarillo-Cisneros, J.; Liu, W.; Ferri, N.; Reuter, K.; Tkatchenko, A. Adsorption Structures and Energetics of Molecules on Metal Surfaces: Bridging experiment and theory. Progr. Surf. Sci. 2016, 91, 72-100.

[14] Wang, Y. Z.; Sun, R. G.; Meghdadi, F.; Leising, G.; Epstein, A. J. Multicolor Multilayer Light-Emitting Devices Based on Pyridine-Containing Conjugated Polymers and para-Sexiphenyl Oligomer. Appl. Phys. Lett. 1997, 74, 3613.

[15] Simbrunner, C. Epitaxial Growth of Sexi-Thiophene and para-Hexaphenyl and its Implications for the Fabrication of Self-Assembled Lasing Nano-Fibres. Semicond. Sci. Technol. 2013, 28, 053001.

[16] Koller, G.; Berkebile, S.; Oehzelt, M.; Puschnig, P.; Ambrosch-Draxl, C.; Netzer, F. P.; Ramsey, M. G. Intra- and Intermolecular Band Dispersion in an Organic Crystal. Science 2007, 317, 351-355.

[17] Hla, S.-W.; Braun, K.-F.; Wassermann, B.; Rieder, K.-H. Controlled Low-Temperature Molecular Manipulation of Sexiphenyl Molecules on Ag(111) Using Scanning Tunneling Microscopy. Phys. Rev. Lett. 2004, 93, 208302.

[18] Braun, K.-F.; Hla.; S.-W. Probing the Conformation of Physisorbed Molecules at the Atomic Scale Using STM Manipulation. Nano Lett. 2005, 5, 73-76.

[19] Oehzelt, M.; Grill, L.; Berkebile, S.; Koller, G.; Netzer, F. P. Ramsey, M. G. The Molecular Orientation of para-Sexiphenyl on $\mathrm{Cu}(110)$ and $\mathrm{Cu}(110) \mathrm{p}(2 \times 1) \mathrm{O}$. ChemPhysChem 2007, 8, 1707-1712.

[20] Chen, W.; Zhang, H. L.; Huang, H.; Chen, L.; Wee, A. T. S. Self-Assembled Organic Donor/Acceptor Nanojunction Arrays. Appl. Phys. Lett. 2008, 92, 193301.

[21] Desiraju, G. R. Hydrogen Bridges in Crystal Engineering: Interactions without Borders. Acc. Chem. Res. 2002, 35, 565-573.

[22] Dunitz, J. D.; Taylor, R. Organic Fluorine Hardly Ever Accepts Hydrogen Bonds. Chem. Eur. J 1997, 3, 89-98.

[23] Shimoni, L.; Glusker. J. P. The Geometry of Intermolecular Interactions in Some Crystalline FluorineContaining Organic Compounds. Structural Chemistry 1994, 5, 283-397. 
[24] Howard, J. A. K.; Hoy, V. J.; O'Hagan, D.; Smith, G. T. How Good is Fluorine as a Hydrogen Bond Acceptor? Tetrahedron 1996, 52, 12613-12622.

[25] de Oteyza, D. G.; García-Lastra, J.M.; Corso, M.; Doyle, B. P.; Floreano, L.; Morgante, A.; Wakayama, Y.; Rubio, A.; Ortega, J. E. Balancing Intermolecular and Molecule-Substrate Interactions in Supramolecular Assemblies. Adv. Funct. Mater. 2009, 19, 259-264.

[26] El-Sayed, A.; Mowbray, D. J.; García-Lastra, J.M.; Rogero, C.; Goiri, E.; Borghetti, P.;Turak, A.; Doyle, B. P.; Dell’Agela, M.; Floreano, L.; et al. Supramolecular Environment-Dependent Electronic Properties of Metal-Organic Interfaces. J. Phys. Chem. C 2012, 116, 4780-4785

[27] Sparenberg, M.; Zykov, A.; Beyer, P.; Pithan, L.; Weber, C.; Garmshausen, Y.; Carlà, F.; Hecht, S.; Blumstengel, S.; Henneberger, F.; et al. Controlling the Growth Mode of para-Sexiphenyl (6P) on ZnO by Partial Fluorination. Phys. Chem. Chem. Phys. 2014, 16, 26084-26093.

[28] Nielsen, C. B.; White, A. J. P.; McCulloch, I. Effect of Fluorination of 2,1,3-Benzothiadiazole. J. Org. Chem. 2015, 80, 5045-5048.

[29] Fischer, F. S. U.; Kayunkid, N.; Trefz, D.; Ludwigs, S.; Brinkmann, M. Structural Models of Poly(cyclopentadithiophene-alt-benzothiadiazole) with Branched Side Chains: Impact of a Single Fluorine Atom on the Crystal Structure and Polymorphism of a Conjugated Polymer. Macromolecules 2015, 48, 3974-3982.

[30] Do, K.; Saleem, Q.; Ravva, M. K.; Cruciani, F.; Kan, Z.; Wolf, J.; Hansen, M. R.; Beaujuge, P. M.; Brédas J.-L. Impact of Fluorine Substituents on $\pi$-Conjugated Polymer Main-Chain Conformations, Packing, and Electronic Couplings. Adv. Mater. 2016, 28, 8197-8205.

[31] Li, W.; Albrecht, S.; Yang, L.; Roland, S.; Tumbleston, J. R.; McAfee, T.; Yan, L.; Kelly, M. A.; Ade, H.; Neher, D.; et al. Mobility-Controlled Performance of Thick Solar Cells Based on Fluorinated Copolymers. J. Am. Chem. Soc. 2014, 136, 15566-15576.

[32] Fei, Z.; Boufflet, P.; Wood, S.; Wade, J.; Moriarty, J.; Gann, E.; Ratcliff, E. L.; McNeill, C. R.. Sirringhaus, H. Kim, J.-S.; et al. An Alkylated Indacenodithieno[3,2-b]thiophene-Based Nonfullerene 
Acceptor with High Crystallinity Exhibiting Single Junction Solar Cell Efficiencies Greater than 13\% with Low Voltage Losses. Adv. Mater. 2018, 30, 1705209.

[33] Garmshausen, Y.; Schwarz, J.; Hildebrandt, J.; Kobin, B.; Pätzel, M.; Hecht, S. Making Nonsymmetrical Bricks: Synthesis of Insoluble Dipolar Sexiphenyls Org. Lett. 2014, 16, 2838-2841. [34] Hla, S.-W. STM Single Atom/Molecule Manipulation and its Application to Nanoscience and Technology. J. Vac. Sci. Technol. B 2005, 23, 1351-1360.

[35] Blum, V.; Gehrke, R.; Hanke, F.; Havu, P.; Havu, V.; Ren, X.; Reuter, K.; Scheffler, M. Ab Initio Molecular Simulations With Numeric Atom-Centered Orbitals. Comput. Phys. Commun. 2009, 180, 2175-2196.

[36] Tkatchenko, A.; Scheffler, M. Accurate Molecular Van Der Waals Interactions from Ground-State Electron Density and Free-Atom Reference Data. Phys. Rev. Lett. 2009, 102, 073005.

[37] Ruiz, V. G.; Liu, W.; Zojer, E.; Scheffler, M.; Tkatchenko, A. Density-Functional Theory with Screened van der Waals Interactions for the Modeling of Hybrid Inorganic-Organic Systems. Phys. Rev. Lett. 2012, 108, 146103.

[38] Tkatchenko, A.; DiStasio, R. A.; Jr.; Car, R.; Scheffler, M. Accurate and Efficient Method for ManyBody van der Waals Interactions. Phys. Rev. Lett. 2012, 108, 236402.

[39] Lifshitz, E. M. The Theory of Molecular Attractive Forces Between Solids. Sov. Phys. JETP. 1956, 2, 73-83.

[40] Schatschneider, B.; Liang, J.-J.; Reilly, A. M.; Marom, N.; Zhang, G.-X.; Tkatchenko, A. Electrodynamic Response and Stability of Molecular Crystals. Phys. Rev. B 2013, 87, 060104.

[41] Perdew, J. P.; Burke, K.; Ernzerhof, M. Generalized Gradient Approximation Made Simple. Phys. Rev. Lett. 1996, 77, 3865.

[42] van Lenthe, E.; Baerends, E. J.; Snijders, J. G. Relativistic Total Energy Using Regular Approximations. J. Chem. Phys. 1994, 101, 9783-9792.

[43] Monkhorst, H. J.; Pack, J. D. Special Points for Brillouin-Zone Integrations. Phys. Rev. B 1976, 13, 5188. 
[44] Liu, W.; Ruiz, V. G.; Zhang, G.-X.; Santra, B.; Ren, X.; Scheffler, M.; Tkatchenko, A. Structure and Energetics of Benzene Adsorbed on Transition-Metal Surfaces: Density-Functional Theory with van der Waals Interactions Including Collective Substrate Response. New J. Phys. 2013, 15, 053046.

[45] Liu W.; Maaß, F.; Willenbockel, M.; Bronner, C.; Schulze, M.; Soubatch, S.; Tautz, F. S.; Tegeder, P.; Tkatchenko, A. Quantitative Prediction of Molecular Adsorption: Structure and Binding of Benzene on Coinage Metals. Phys. Rev. Lett. 2015, 115, 036104.

[46] Charge-Carrier Mobility in Organic Crystals; Farchioni, R.; Grosso, G.; Ed.; Springer Berlin Heidelberg, 2001; pp. 283-326.

[47] Hirshfeld, F. L.; Bonded-Atom Fragments for Describing Molecular Charge Densities. Theoretica chimica acta 1977, 44, 129-138.

[48] Sadhukhan, M.; Tkatchenko, A. Long-Range Repulsion Between Spatially Confined van der Waals Dimers. Phys. Rev. Lett. 2017, 118, 210402.

[49] Müllegger, S. Salzmann, I.; Resel, R.; Hlawacek, G.; Teichert, C.; Winkler, A. Growth Kinetics, Structure, and Morphology of para-Quaterphenyl Thin Films on Gold(111). J. Chem. Phys. 2004, $121,2272-2277$.

[50] Lukas, S.; Witte, G.; Wöll, Ch. Novel Mechanism for Molecular Self-Assembly on Metal Substrates: Unidirectional Rows of Pentacene on Cu(110) Produced by a Substrate-Mediated Repulsion. Phys. Rev. Lett. 2001, 88, 028301.

[51] Stadler, C.; Hansen, S.; Kröger, I.; Kumpf, C.; Umbach, E. Tuning Intermolecular Interaction in LongRange-Ordered Submonolayer Organic Films Nat. Phys. 2009, 5, 153-158.

[52] Duhm, S.; Bürker, C.; Niederhausen, J.; Salzmann, I.; Hosokai, T.; Duvernay, J.; Kera, S.; Schreiber, F.; Koch, N.; Ueno, N.; et al. Pentacene on Ag(111): Correlation of Bonding Distance with Intermolecular Interaction and Order. ACS Appl. Mater. Interfaces 2013, 5, 9377-9381.

[53] Wagner, C.; Kasemann, D.; Golnik, C.; Forker, R.; Esslinger, M.; Müllen, K.; Fritz, T. Repulsion Between Molecules on a metal: Monolayers and Submonolayers of hexa-peri-Hexabenzocoronene on Au(111) Phys. Rev. B 2010, 81, 035423. 
[54] Seidel, C.; Ellerbrake, R.; Gross, L.; Fuchs, H. Structural Transitions of Perylene and Coronene on Silver and Gold Surfaces: A Molecular-Beam Epitaxy LEED Study. Phys. Rev. B 2001, 64, 195418.

[55] Thussing, S.; Jakob, P. Structural and Vibrational Properties of CuPc/Ag(111) Ultrathin Films. J. Phys. Chem. C 2016, 120, 9904-9913.

[56] Song, Z.; Schultz, T.; Ding, Z.; Lei, B.; Han, C.; Amsalem, P.; Lin, T.; Chi, D.; Wong, S. L.; Zheng, Y. J.; et al. Electronic Properties of a 1D Intrinsic/p-Doped Heterojunction in a 2D Transition Metal Dichalcogenide Semiconductor. ACS Nano 2017, 11, 9128-9135.

[57] Tyson, W. R.; Miller, W.A. Surface Free Energies of Solid Metals: Estimation from Liquid Surface Tension Measurements. Surf. Sci. 1977, 62, 267-276.

[58] Li, R.; Zhang, X.; Dong, H.; Li, Q.; Shuai, Z.; Hu, W. Gibbs-Curie-Wulff Theorem in Organic Materials: A Case Study on the Relationship between Surface Energy and Crystal Growth. Adv. Mater. 2016, 28, 1697-1702.

[59] Arras, E.; Seitsonen, A. P.; Klappenberger, F.; Barth, J. V. Nature of the Attractive Interaction Between Proton Acceptors and Organic Ring Systems Phys. Chem. Chem. Phys. 2012, 14, 15995-16001.

[60] Palma, C.-A.; Björk, J.; Klappenberger, F.; Arras, E.; Kühne, D.; Stafström, S.; Barth, J. V. Visualization and Thermodynamic Encoding of Single-Molecule Partition Function Projections Nat. Comm. 2015, 6, 6210.

[61] Thalladi, V. R.; Weiss, H.-C.; Bläser, D.; Boese, R.; Nangia, A.; Desiraju, G. R. C-H‥F Interactions in the Crystal Structures of Some Fluorobenzenes J. Am. Chem. Soc. 1998, 120, 8702-8710.

[62] Thakur, T. S.; Kirchner, M. T.; Bläser, D.; Boese, R.; Desiraju. G. R. C-H $\cdots$ F-C Hydrogen Bonding in 1,2,3,5-Tetrafluorobenzene and other Fluoroaromatic Compounds and the Crystal Structure of Alloxan Revisited. CrystEngComm, 2012, 12, 2079-2085. 
TOC Graphic

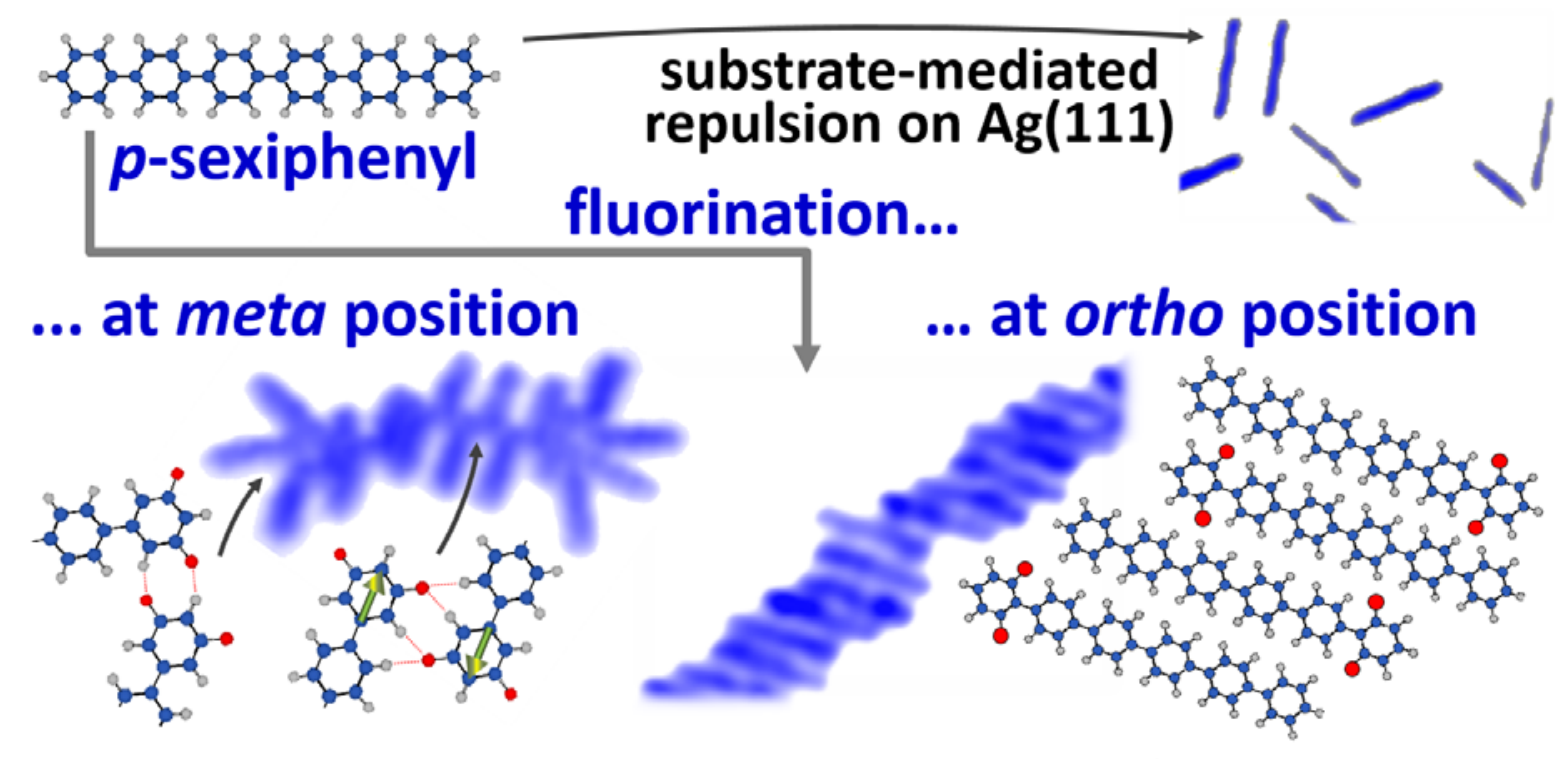

specifics of $\mathrm{H} \cdots \mathrm{F}$ bonds + molecular polarity $\Rightarrow$ lateral overlap 